\title{
Hepatic Venous Duplex as an Alternative Non- invasive Diagnostic Tool for Diagnosis of Pulmonary Hypertension.
}

\author{
MD. HARISUL HOQUE, SM MUSTAFAZAMAN, KHURSHID AHMED, SAJAL KRISNA BANERJEE \\ MD. FAISAL IBNE KABIR, NILUFAR FATEMA
}

Department of Cardiology, Bangabandhu Sheikh Mujib Medical University, Dhaka.

Address of Correspondence : Dr. Nilufar Fatema, Consultant, Department of Cardiology, Bangabandhu Sheikh Mujib Medical University, Dhaka. Email: nilufar.fatema@gmail.com

\begin{abstract}
:
Pulmonary hypertension is a hemodynamic disorder defined by abnormally high pulmonary artery pressure that affects the arteries in your lungs and the right side of your heart. In this study, hepatic venous duplex will be done to diagnose and quantify the PH. So that Patients can avoid unnecessary invasive right heart catheterization. This practical demonstration is the key to enrich our experience and knowledge in the field of PH. Objectives of this study was to assess PH status by Hepatic venous Duplex (HVD) as well by right heart catheterization and to compare them. This study was conducted in the Department of Cardiology, BSMMU, Shahbagh, Dhaka extending from July 2018 to December 2019. Total 100 (One hundred) subjects were enrolled in this study. It was an Observational study and includes the subjects between 18 years to 45 years of age. Results of this study shows very close proximity to that of Right heart catheterization. Hemodynamic changes in Hepatic venous duplex study could be used as an alternative diagnostic tool for evaluating moderate to severe pulmonary hypertension. This method could counteract the weakness of the currently used diagnostic methods and improve the accuracy of assessing pulmonary hypertension when combined with other methods.
\end{abstract}

Key words: Pulmonary Hypertension, Right heart catheretization, Hepatic Venous Duplex

University Heart Journal 2020; 16(2): 86-91

\section{Introduction:}

Pulmonary hypertension is a hemodynamic disorder defined by abnormally high pulmonary artery pressure that affects the arteries in your lungs and the right side of your heart. It can occur in numerous diseases and clinical situations. In one form of pulmonary hypertension, tiny arteries in your lungs, called pulmonary arterioles, and capillaries become narrowed, blocked or destroyed. This makes it harder for blood to flow through your lungs, and raises pressure within your lungs' arteries. As the pressure builds, your heart's lower right chamber (right ventricle) must work harder to pump blood through your lungs, eventually causing your heart muscle to weaken and fail. Some forms of pulmonary hypertension are serious conditions that become progressively worse and are sometimes fatal. Although some forms of pulmonary hypertension aren't curable, treatment can help lessen symptoms and improve your quality of life. The causes of pulmonary hypertension are classified into 5 major groups: arterial, due to left heart disease, due to lung disease and /or hypoxemia, chronic thromboembolic, with unclear and/ or multifactorial mechanisms. For the past 3 decades, there has been an increased diagnosis of pulmonary hypertension $(\mathrm{PH})$ in clinical practice. It is likely that this rise in $\mathrm{PH}$ diagnoses is attributable to multiple factors, including increased awareness by clinicians, the routine use of diagnostic tools such as Doppler echocardiography. Now a day, life expectancy of pulmonary hypertension patients has increased due to availability and marketing of the many PH-specific drugs. ${ }^{1}$

Severe tricuspid regurgitation (TR) develops in $10-30 \%$ of patients with pulmonary hypertension $(\mathrm{PH})$ and early death in $1 / 3$ such patients within one year. ${ }^{2-3}$ Patients with tricuspid regurgitation may present initially with vague abdominal symptoms and elevated liver enzymes. Pulmonary artery systolic pressure is calculated by adding right atrial pressure with peak TR gradient. Sometime, it is difficult to get tricuspid regurgitation gradient. In the absence of diagnostic sonographic findings, patients may be subjected to an unnecessary invasive right heart catheterization for an accurate diagnosis. The right heart catheterization is the gold standard investigation for diagnosing the pulmonary hypertension. The Doppler flow 
pattern in the hepatic venous flow was triphasic, reflecting right atrial pressures and right ventricular dysfunction, allows prediction of increased right atrial pressure and right ventricular dysfunction in patients with right ventricular infarction. In a study, patients with sonographic signs of congestive heart failure, duplex Doppler sonography of the hepatic vein may be helpful in the diagnosis of one of the causes of liver dysfunction and tricuspid regurgitation. ${ }^{4}$

In this study, hepatic venous duplex will be done to diagnose and quantify the PH. So that Patients can avoid unnecessary invasive right heart catheterization. This practical demonstration is the key to enrich our experience and knowledge in the field of PH.

\section{Objectives}

1. To assess PH status by Hepatic venous Duplex (HVD).

2. To assess $\mathrm{PH}$ status by right heart catheterization.

3. To compare the HVD study with the right heart catheterization findings.

\section{Materials and Methods:}

Study place: Department of Cardiology, BSMMU, Shahbagh, Dhaka

Study population: Patients who are suspected case of pulmonary hypertension admitted in cardiology department of BSMMU and the patient who are attending at the OPD of BSMMU as well were included in this study after obtaining consent.

\section{Study population}

a) Sample Size: 100 (one hundred) patients

b) Age: Between 18 to 45 yrs.

Study period: July 2018 to December 2019.

Study design: Observational study

\section{Selection criteria}

-Inclusion criteria: Pulmonary hypertension due to any cause

-Exclusion criteria: The patient who is not willing to participate in the study.

\section{Working Defination of Pulmonary Hypertension}

According to MAYO Clinic, Pulmonary hypertension is a type of high blood pressure that affects the arteries in lungs and the right side of heart. In one form of pulmonary hypertension, tiny arteries in lungs, called pulmonary arterioles, and capillaries become narrowed, blocked or destroyed. This makes it harder for blood to flow through lungs, and raises pressure within lungs' arteries. As the pressure builds, heart's lower right chamber (right ventricle) must work harder to pump blood through the lungs, eventually causing the heart muscle to weaken and fail. Some forms of pulmonary hypertension are serious conditions that become progressively worse and are sometimes fatal. Although some forms of pulmonary hypertension aren't curable, treatment can help lessen symptoms and improve the quality of life.

\section{Method of study}

Colour Doppler echocardiographies were done in 100 study population to detect cause and grading of pulmonary hypertension by GE Echocardiography machine. For the quantification of pulmonary hypertension diagnosis, Tricuspid regurgitation gradient was measured. After detectection of TR and subsequently estimation of TR gradient are measured and RA pressure are addet with this result. Usually PH was quantified by this way. Hepatic venous Duplex study was done by pulse wave by GE Vivid 7 Ultrasound Machine with a transducer M4S. Single operator has performed the duplex ultrasound without having background knowledge about patients group. Image was taken in B-mode along with spectral Doppler and color flow imaging. The cursor was placed in $1.0 \mathrm{~cm}$ away from the junction of IVC and hepatic vein by pulse wave. Spectral flow was seen and measurement will be taken. 3 consecutive spectral displays were taken for the patient with sinus rhythm and 5 consecutive spectral displays were taken for patients with atrial fibrillation. Average of these measurements was taken. Hepatic venous flow patterns included doublepeaked flow signals toward the right atrium, and the relationship between systolic (S) and diastolic flow velocities (D) was expressed as the velocity ratio [S/ $(\mathrm{S}+\mathrm{D})$ ]. A reversed flow during atrial systole was expressed as an "A wave" and that between the S and D waves, as an "O wave". 5 Hepatic venous flow was measured at peak velocity and velocity time integrals (VTI) of the systolic (S), diastolic (D) and atrial (a) contraction waves. The fraction of systolic filling was calculated: VTI S/VTI S + VTI D. The pressure half-time of pulmonary regurgitate flow (PHT IP) was measured. The A wave was increased in cases with pulmonary hypertension and A wave velocity in the hepatic vein correlated with systolic pulmonary artery pressure. In cases with tricuspid regurgitation, reversed flows were detected during ventricular systole both in normal sinus rhythm and in atrial fibrillation ${ }^{5}$. PH was graded by finding of alteration of hepatic venous duplex pattern. These hepatic venous duplex findings were compared with right heart catheter findings and statistical analysis was done.

Hepatic venous flow alteration in Tricuspid Regurgitation (TR) by Hepatic venous duplex: In Mild TR, there is no effect. In Moderate TR, decrease in ' $\mathrm{S}$ ' and in Severe TR, ' $\mathrm{S}$ ' is replaced by SR (retrograde systolic reversal) 
HV flow is altered in TR

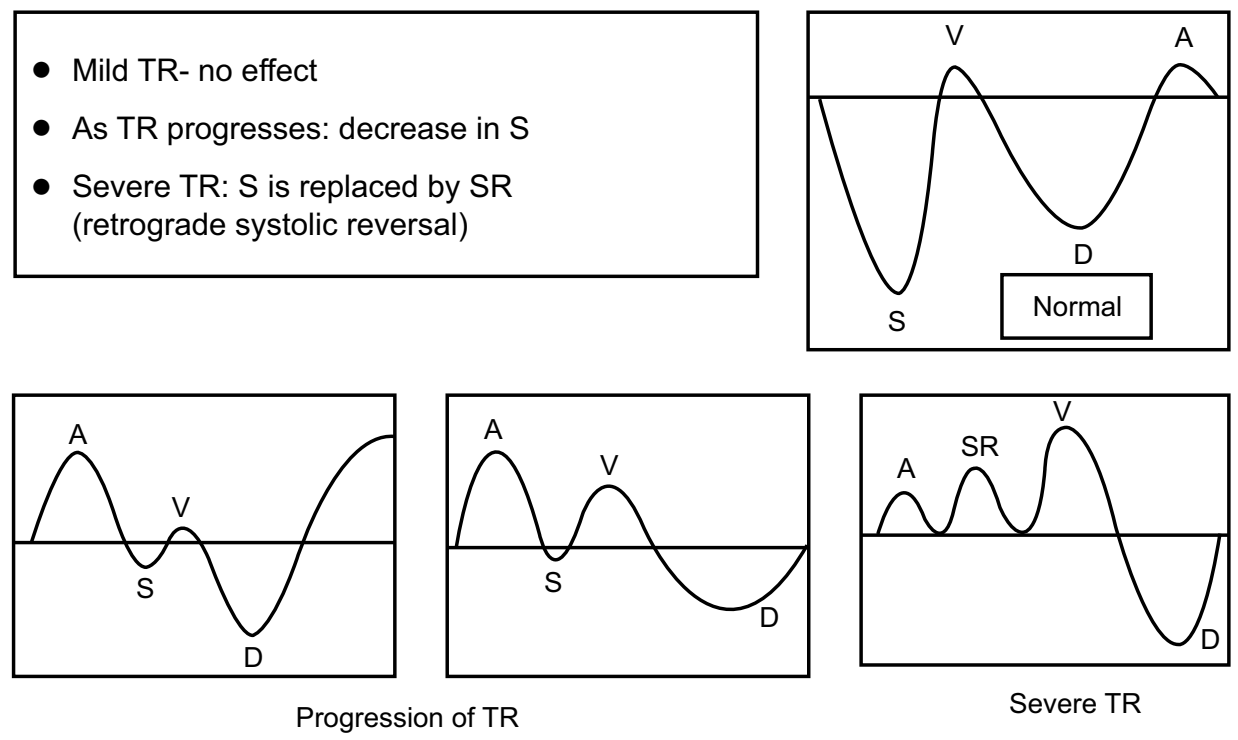

Fig.-1: Changes of hepatic venous Doppler flow with the progression of TR.

These patients went for right heart catheterization later. Then data was compared to identify the pulmonary artery systolic pressure and quantification of pulmonary hypertension was done in both by conventional Color Doppler Echocardiography, hepatic venous duplex and right heart catheterization.

\section{H. Data collection Procedure}

100 patients were recruited in this study. All data were noted in pre formatted data sheet as approved by Institutional Review board of BSMMU. All relevant Echo report and print outs were kept for analysis.

\section{Statistical Analysis:}

Parametric data were evaluated by independent $t$ test and categorical data were evaluated by Chi square test. Significance were ascribed as $\mathrm{p}$ value $<0.05$. Multivariate regression analysis has been done by SPSS 16.0 for windows software. Categorical data has been expressed in percentage or number. Parametric datas are expressed in mean +- SD.

\section{Results:}

Among 100 pulmonary hypertension patients, 34 had mild, 33 had moderate and rest 33 had severe pulmonary hypertension in color Doppler echocardiography. Hepatic venous duplex was done in these 100 patients. In case of hepatic venous duplex, 19 had mild PH of 34 Mild PH patients. Rest of 15 patients who had been detected as mild PH by color Doppler echo went to moderate PH group by hepatic venous duplex study which is similar of right heart catheter findings. Echo diagnosed 33 moderate $\mathrm{PH}$ patients had been interrogated by hepatic venous duplex, 10 patients found to have severe $\mathrm{PH}$, rest remain in moderate $\mathrm{PH}$ group. These findings also have similarity with right heart catheter findings. And the third group: 33 severe PH by Echo, 43 were detected as severe by hepatic venous duplex and 46 patients were confirmed as severe by right heart catheter. Invasive catheter findings had similarity with noninvasive hepatic venous duplex. 19 patients showed normal four phagic waves in hepatic venous duplex where deep $\mathrm{S}$ in between small A wave and small $\mathrm{V}$ wave and that patients have mild pulmonary hypertension. In 38 moderate $\mathrm{PH}$ patients, $\mathrm{S}$ wave became very small in between large $A$ wave and small $V$ wave are seen. And 43 Severe $\mathrm{PH}$ have no $\mathrm{S}$ wave but reverse $\mathrm{S}$ wave (SR) was seen in between small A wave and large $V$ wave.

Table-I

Number of patients with PH detected by different modalities $(N=100)$

\begin{tabular}{lccc}
\hline Status of Pulmonary Hypertension by Color doppler & Hepatic duplex & Right heart catheter & p-value \\
Echo (TR gradient+ RA Pressure) & & 17 & $\mathrm{P}=0.083$ \\
\hline Mild $(\mathrm{n}=34)$ & 19 & 37 & $\mathrm{P}=0.04$ \\
Moderate $(\mathrm{n}=33)$ & 48 & 46 & $\mathrm{P}=0.05$ \\
Severe $(\mathrm{n}=33)$ & 43 & \\
\hline
\end{tabular}




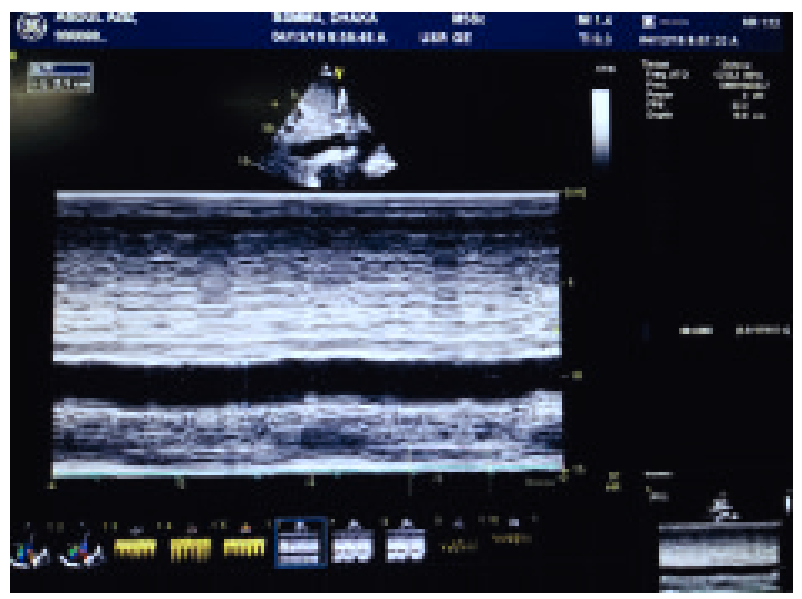

Fig.-2: Severe PH by TR gradient and IVC findings in Color Doppler Echo in patient ' $X$ '

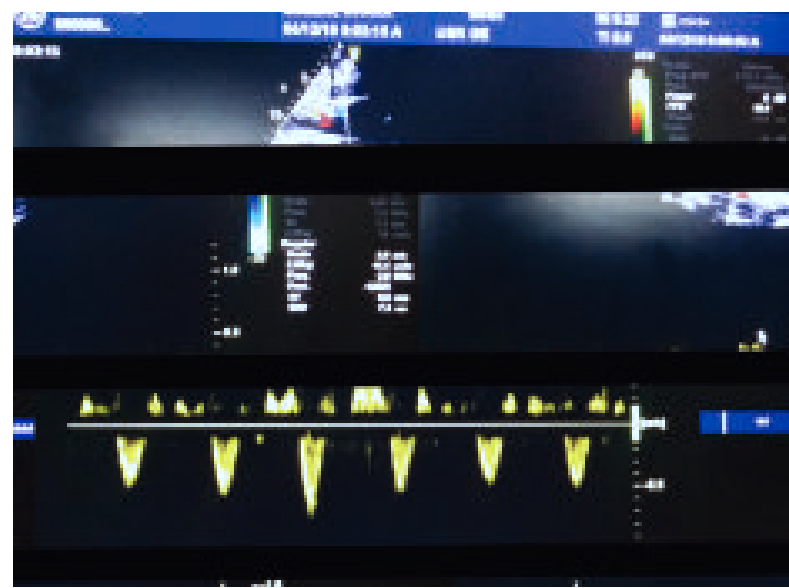

Fig.-3: Changes of Hepatic venous duplex in Severe PH in ' $X$ ' patient

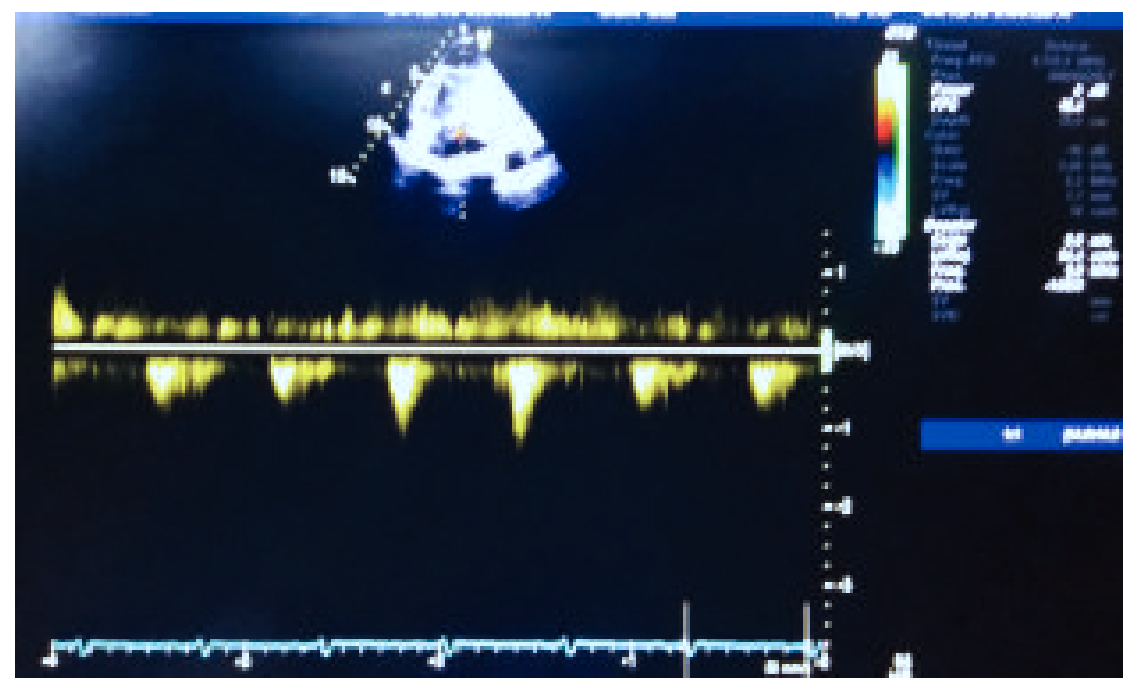

Fig.-4: Changes of Hepatic venous duplex in Moderate PH in ' $Y$ ' patient

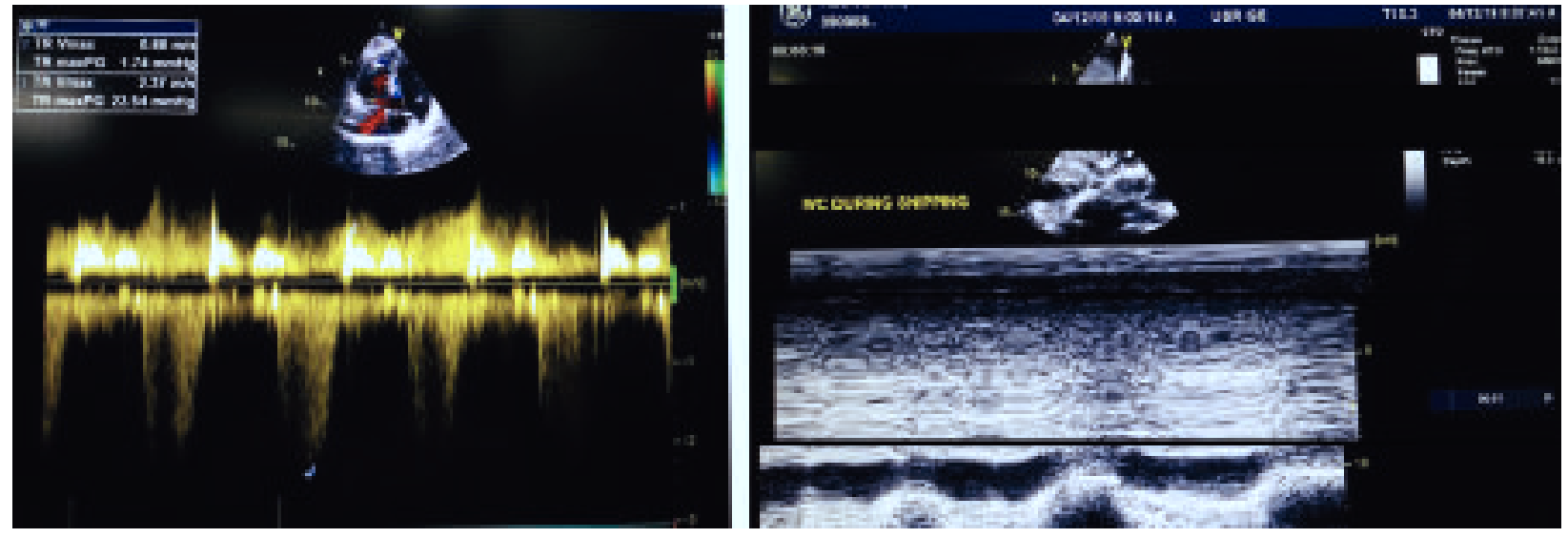

Fig.-5: Mild PH, Panel A: TR gradient, Panel B: IVC finding during snipping in Patient ' $Z$ ' 


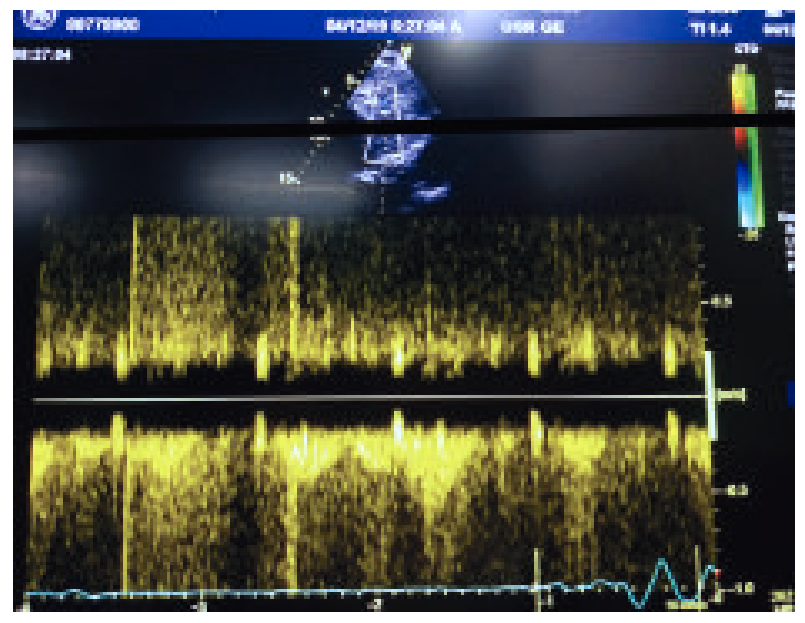

Fig.-6: Changes in Hepatic venous duplex in mild PH in patient ' $Z$ '.

\section{Discussions:}

The aim of this study was to test the hypothesis that Doppler study of hepatic venous flow, reflecting right atrial pressures which allows prediction of increased right atrial pressure in patients with pulmonary hypertension. The flow pattern of the right hepatic vein was classified as triphasic, biphasic and monophasic. There was a correlation observed between Doppler parameters of hepatic venous flow and hemodynamic data from right heart catheterization, in particular right atrial pressure and pressure wave form. Moreover, statistically no significant difference was observed between the two investigations in respect to the Doppler parameters derived from hepatic venous flow and right heart catheterization. Karabulut $\mathrm{N}$ et al shows in a study that in normal individual about $99 \%$ have triphasic and $1 \%$ has biphagic flow pattern in hepatic vein. ${ }^{6}$ There are little changes in hepatic venous flow in case of mild pulmonary hypertension $85 \%$ patients are triphasic and only $15 \%$ patients are biphagic in this study. On the other hand, the results confirmed good diagnostic performance of Doppler analysis of hepatic venous flow in patients with severe pulmonary hypertension: sensitivity $80 \%$, specificity $83 \% .{ }^{6}$ Doppler analysis of hepatic venous flow allows assessment of right atrial pressure in pulmonary hypertension patients. Alyse $\mathrm{M}$ et al showed an important finding of their study was that Doppler assessment of hepatic venous velocities demonstrates reduced or negative velocities in the first half of diastole in children with right ventricular hypertension. The other hepatic venous waveform change occurred during end-diastole and atrial contraction. At that time amplitude of reversed blood velocity in the hepatic vein increased directly with increased right ventricular pressure, but the magnitude of increase was not clinically significant. ${ }^{7}$ These findings have similarity with this present study findings. Hepatic venous velocities have been reduced or negative velocity was noted in case of moderate to severe pulmonary hypertension. Dan Dan Sun et al. have shown in their study that in control group, there were 18 patients with four phagic waves ( $\mathrm{S}$ wave, $\mathrm{D}$ wave, $\mathrm{A}$ wave and $\mathrm{V}$ wave) and 10 patients with tri-phagic waves (s wave, D wave and $A$ wave). In pulmonary hypertension group, there were 13 patients with four phagic waves ( $\mathrm{S}$ wave, D wave, A wave and $\mathrm{V}$ wave), 28 patients with triphagic waves ( $\mathrm{S}$ wave, D wave and A wave), 9 patients with bi-phagic waves (S wave and A wave) and 2 patients with mono-phagic flat waveform. In their study, they only analyzed the normal basic waveform of hepatic vein, including two antegrade waves ( $\mathrm{S}$ wave and $\mathrm{D}$ wave) and one retrograde (A wave). ${ }^{8}$ In this study, 19 patients showed normal four phagic waves in hepatic venous duplex where deep $\mathrm{S}$ in between small $\mathrm{A}$ wave and small $\mathrm{V}$ wave and that patients have mild pulmonary hypertension. In 38 moderate $\mathrm{PH}$ patients, $\mathrm{S}$ wave became very small in between large A wave and small $V$ wave are seen. And 43 Severe $P H$ have no $S$ wave but reverse $\mathrm{S}$ wave $(\mathrm{SR})$ was seen in between small $\mathrm{A}$ wave and large $\mathrm{V}$ wave.

\section{Conclusions:}

Hemodynamic changes in Hepatic venous duplex study could be used as an alternative diagnostic tool for evaluating moderate to severe pulmonary hypertension. This method could counteract the weakness of the currently used diagnostic methods and improve the accuracy of assessing pulmonary hypertension when combined with other methods.

\section{Ethical Issues}

There were minimum physical, psychological, social and legal risk during history taking, physical exam and investigation. Proper safety measures were ensured in every steps of the study. Only research personnel were allowed to access data. Ethical clearance has been obtained from the authority of BSMMU to undertake the present study. According to Helsinki Declaration for Medical Research involving Human Subjects 1964, all the patients were informed about the study design, the underlying hypothesis and the right for the participants to withdraw themselves from the study at any time, for any reason, what so ever which was not hamper the standard duty of care anyway. Informed written consent was obtained from each subject who was voluntarily provide consent to 
participate in the study. The ethical issues was addressed accordingly,

1) Strict confidentiality and security of data related to patient was maintained. The presentation of data and information related to patient was documented anonymously.

2) The data analysis was completed on subjects who complete the study according to protocol after recruitment of subjects with valid informed consent.

3) As the procedures were non-invasive and invasive, there was risk or safety concern to each patient and researcher.

4) There was no potential conflict of interest in this study and it was entirely an academic research project.

5) The research was routine and customary for this hospital. Therefore, there was no additional burden on the patient or on the service providers.

\section{Declaration of interests}

This project has been done with the fund obtained from Bangabandhu Sheikh Mujib Medical University Research Allocation for the Fiscal year 2018-19.

\section{Acknowledgements}

The authors would like to thank the patients who participated in this study and their family members, as well as other investigator and stuff at all of Cardiology department of BSMMU.

\section{References:}

1. McGoon MD, Benza RL, Escribano-Subias P, Jiang X, Miller DP, Peacock AJ, Pepke-Zaba J, Pulido T, Rich S, Rosenkranz S, Suissa S, Humbert M. Pulmonary arterial hypertension: epidemiology and registries. J Am Coll Cardiol. 2013; 62(25 Suppl):D51-D59.

2. Nath J, Foster E, Heidenreich PA. Impact of tricuspid regurgitation on long-term survival. J Am Coll Cardiol. 2004; 43: 405-09.

3. Koelling TM, Aaronson KD, Cody RJ, Bach DS, Armstrong WF. Prognostic significvance of mitral regurgitation and tricuspid regurgitation in patients with left ventricular systolic dysfunction. Am Heart J. 2002; 144:524-29.

4. Vinsonneau C, Carrat F, Boccara F, Buyukoglu B, Lamisse N, Cohen A. Does Doppler analysis of hepatic venous flow predict increased right atrial pressure and right ventricular dysfunction. Circulation; 2016; 101: 347-52

5. Sakoda S, Mitsunami K, Kinoshita M. Evaluation of hepatic venous flow patterns using a pulsed Doppler technique. J Cardiol. 1990;20(1):193-208

6. Karabulut N, Kazil S, Yagci B, Sabir NEur Radiol. Doppler waveform of the hepatic veins in an obese population. Eur Radiol. 2004; 14 (12): 2268-72

7. Alyse M, BenderBA, Jonathan A, BaronBA, Stanley J, GoldbergMD, Nancy J, BouherRDCS. Hepatic vein velocities in children with right ventricular hypertension. The American Journal of Cardiology; 1995; 75 (14): 971-73

8. Dan Dan Sun, Chuan Ju Hou, Li Jun Yuan, Yun You Duan, Ying Hou, FangPing Zhou. Hemodynamic changes of middle hepatic vein in patients with pulmonary hypertension using echocardiography. Journal.pone. 0121408, March; 2015 